\title{
Ähnlichkeit und Kontrafakt
}

Ökonomische Praktiken im nichtjüdischen und jüdischen deutschen Bürgertum am Beispiel von Gustav Freytags Soll und Haben

Similarité et contrafactualité. Les pratiques économiques dans la bourgeoisie allemande non juive et juive à l'exemple du roman Soll und Haben de Gustav

Freytag

Similarity and Counterfact. Economic Practices in the Non-Jewish and Jewish Bourgeoisie, Exemplified by the Novel Soll und Haben by Gustav Freytag

\section{Nicole COLIN}

\section{(2) OpenEdition}

\section{Journals}

Édition électronique

URL : https://journals.openedition.org/ceg/7254

DOI : $10.4000 /$ ceg. 7254

ISSN : 2605-8359

Éditeur

Presses Universitaires de Provence

\section{Édition imprimée}

Date de publication : 25 septembre 2019

Pagination : 107-119

ISBN : 979-10-320-0234-6

ISSN : 0751-4239

Référence électronique

Nicole COLIN, „Ähnlichkeit und Kontrafakt", Cahiers d'Études Germaniques [Online], 77 | 2019, Online erschienen am: 25 März 2021, abgerufen am 16 Juni 2021. URL: http://journals.openedition.org/ceg/ 7254 ; DOI: https://doi.org/10.4000/ceg.7254 


\section{Ähnlichkeit und Kontrafakt Ökonomische Praktiken im nichtjüdischen und jüdischen deutschen Bürgertum am Beispiel von Gustav Freytags Soll und Haben}

Nicole COLIN

Aix-Marseille Université / ECHANGES (4236)

Die Frage nach Identität und Alterität spielt in trans- und interkulturellen Untersuchungen eine zentrale Rolle. Dabei wird in der Regel auf Differenzerfahrungen rekurriert, ${ }^{1}$ die im allgemeinen Verständnis eine potenzielle Quelle für Konflikte darstellen und sich in Beschreibungen oft in Form von Stereotypen verhärten. Dieser Mechanismus lässt sich auch in den Darstellungen des Verhältnisses zwischen Juden und Nichtjuden in Deutschland bestätigen, gleich ob es sich um Abhandlungen oder literarische Zeugnisse handelt, in denen gemeinhin vor allem die Unterschiede der Entwicklung der beiden Gruppen akzentuiert werden. Todd Samuel Presner konstatiert, das "moderne" deutschjüdische Verhältnis beginne mit einem „slash", einem „cut", wobei die Trennung der Begriffe ,deutsch' und ,jüdisch' jedoch ambivalent sei: So könne diese als Gegensatz, Gemeinsamkeit oder auch Wahl interpretiert werden; letztlich besäße die in der Trennung bezeichneten Unterscheidung jedoch die Eigenschaft einer ungelösten Spannung, einer Wechselbeziehung, die sich nicht in einen dritten Begriff transformieren ließe. ${ }^{2}$ Laut Pressner sind die Begriffe deutsch und jüdisch gegenseitig "kontaminiert", sie überschneiden sich und werden unscharf. ${ }^{3}$

1. Dies gilt im Übrigen auch, wenn Fremdheit nicht als Eigenschaft, sondern als Konstrukt bzw., wie beispielsweise bei Alois Hahn, als Zuschreibung und "Definition einer Beziehung" verstanden wird. Vgl. Alois Hahn, „Die soziale Konstruktion des Fremden“, in Walter M. Sprondel (Hrsg.), Die Objektivität ihrer Ordnungen und ihre kommunikative Konstruktion, Frankfurt a. M., 1994, S. 140.

2. "German/Jewish modernity begins with the slash, the cut, the decision, the divider. The separatrix refers to the line between the two words German and Jewish, the cut that separates them. The meaning of the separatrix is ambiguous: it may locate an opposition, as in German versus Jewish, it may signify simultaneity, as in both German and Jewish, and it may call upon a choice, as in German or Jewish. At the same time that the separatrix announces a kind of distinction, the relationship between the distinguished terms is characterized by an unresolved tension, a back-and-forth that is never subdued or sublated into a third term." (Todd Samuel Presner, Mobile Modernity. German, Jews, Trains, New York, Columbia UP, 2007, S. 3-4)

3. " [W]e find the two terms consistently, contaminated' by one another. They overlap; they become blurred" (ibid., S. 4). 
Eine solch unscharfe Spannung ist im Rahmen der kulturellen Differenztheorie mit Begriffen wie Identität und Alterität tatsächlich nicht zu fassen. Im Gegensatz dazu verspricht ein Rückgriff auf den vagen, in Kulturtransferstudien oft vernachlässigten Begriff der Ähnlichkeit eine angemessenere Annäherung an das Problemfeld. Das Konzept - mit Dorothee Kimmich und Anil Bhatti verstanden als "kulturtheoretisches Paradigma"4 - bietet sich auch darum an, weil das letztlich auf Identität rekurrierende Differenzdenken, das im positiven Sinne durchaus dazu dienen könnte, die Konstruktion kultureller Hierarchien sichtbar werden zu lassen, sich als recht anfällig für ideologischen Missbrauch erweist. Wie Albrecht Koschorke bemerkt, hat die „Wertschätzung von Heterogenität, Differenz und Hybridität" in den Konzepten der modernen Kulturwissenschaften, die „entscheidende Schwäche, dass sie nur die Gewichtung innerhalb von mächtigen Dichotomien umkehrt, aber das dichotomische Schema an sich unangetastet lässt." ${ }^{5}$ Die antagonistische Gegenüberstellung konstruiert scharfe Unterscheidungen zwischen kulturellen Entitäten, die sich in der Regel gruppenspezifisch oder individuell viel ausdifferenzierter präsentieren, als es das Denken in Kategorien von Identität und Alterität erlaubt. Zudem birgt die (angeblich) präzise Definition der Grenzen in den Analysen transkultureller Phänomene ein nicht zu unterschätzendes Konfliktpotenzial. Im Unterschied dazu kann Ähnlichkeit, Koschorke folgend, als eine "Kategorie der Entdramatisierung" verstanden werden. ${ }^{6}$

Natürlich fußt auch Ähnlichkeitsdenken auf Vergleichen und arbeitet mit Abgrenzung, aber eben in einer unscharfen, nicht exakten Weise. Wie Dorothee Kimmich betont, impliziert Ähnlichkeit "qualitative Nähe“ und kann „als,Figur des Kontinuierlichen', Übergänglichen“ bezeichnet werden:

\footnotetext{
Sie bedarf zwar der Markierung von Differenzen, stellt aber nie einen Bruch oder Gegensatz dar. Im Konzept der Ähnlichkeit können Evolution, Wandel und Metamorphose gedacht werden. Aber auch Selbstverlust, Anpassungsdruck und Assimilation lassen sich mit Ähnlichkeitsmodellen beschreiben. ${ }^{7}$
}

Im Kontext deutsch-jüdischer Beziehungen erscheint das Prinzip darum interessant, weil es einen direkten Bezug zu „Praktiken des intendierten, gewollten,Ähnlichmachens " erlaubt. $^{8}$ Das vage, oft nicht genau zu bestimmende Spannungsfeld zwischen freiwilliger und erzwungener Akkulturation besitzt gerade für den jüdischen Emanzipationsprozess im 19. Jahrhundert in Deutschland eine herausragende Bedeutung. Eine Orientierung am Paradigma der Ähnlichkeit ermöglicht insofern nuancierte Erkenntnisse über das Wechselverhältnis zwischen nicht-jüdischer Mehrheitsgesellschaft und jüdischer

4. Anil Bhatti, Dorothee Kimmich (Hrsg.), Ähnlichkeit. Ein kulturtheoretisches Paradigma, Konstanz, Konstanz UP, 2015.

5. Albrecht Koschorke, "Ähnlichkeit. Valenzen eines post-postkolonialen Konzepts", in Bhatti, Kimmich, Ähnlichkeit, S. 35.

6. Ibid., S. 36.

7. Dorothee Kimmich, „Ähnlichkeit in der Wissensgeschichte“, in Bhatti, Kimmich, Ähnlichkeit, S. 14.

8. Ibid. 
Minorität. Es erlaubt, die Dynamik der komplexen Spannung zwischen jüdischer Integration und gleichzeitiger Exklusion zu berücksichtigen, die sich einer präzisen Beschreibung oft entzieht. Wie Koschorke betont, ist Ähnlichkeit

\begin{abstract}
anders als Identität, mit einer gewissen Intransparenz gut verträglich; sie ist eine adäquate Wahrnehmungsweise, wo Menschen sich nur partial begegnen, in fluiden Beziehungen, Netzwerken, Formen der Teil-Inklusion. Sie bezeichnet einen Modus des zwischenmenschlichen Umgangs unter den Vorzeichen einer auf genügsame Weise unvollständigen Information. ${ }^{9}$
\end{abstract}

Aus genannten Gründen möchte ich in den folgenden Überlegungen den Weg des Denkens in den Kategorien von Identität und Alterität verlassen und stattdessen als methodische Grundlage das Prinzip der Ähnlichkeit wählen, das nicht auf eine "Harmonisierung oder Nivellierung von Unterschieden" zielt, sondern, wie Kimmich betont, "ein subversives Potential“ besitzt, „postulierte Antagonismen [...] als Ideologie [zu] entlarven." ${ }^{10}$

Den Ausgangspunkt meiner Betrachtung stellt ganz allgemein die Annahme dar, dass die Exklusion der jüdischen Minderheit im 19. Jahrhundert in Deutschland an sich keinen Beleg für ihre Andersartigkeit darstellt. Im Gegenteil handelt es sich bei den bekannten Gegenbildern oft um kontrafaktische Konstrukte, welche die oft viel deutlicheren Ähnlichkeiten beider Gruppen bewusst unterminieren bzw. zu kaschieren trachten. Dabei soll der Prozess der Akkulturation bzw. Assimilation des jüdischen Bürgertums hier nicht bewertet oder hinterfragt, sondern neutral als Kulturtransfer und Grundlage einer durchaus produktiven Verflechtungsgeschichte verstanden werden, in der die gesellschaftliche Position der Akkulturierten trotz eines hohen Grades an Integrationsbereitschaft dennoch prekär blieb.

Der Beitrag gliedert sich in zwei Teile: Zunächst werden die allgemeinen Bedingungen der bürgerlichen Emanzipation im 19. Jahrhundert und die kulturellen und sozialen Praktiken des assimilierten jüdischen Bürgertums in Deutschland im Blick auf seine Ähnlichkeiten mit der nichtjüdischen Mehrheitsgesellschaft dargestellt. Dabei ist insbesondere ein Fokus auf die Aspekte Arbeit und Bildung, respektive ökonomisches und symbolisches Kapital, zu legen, die zentrale Elemente des bürgerlichen Selbstverständnisses in beiden Gruppen bildeten. In einem zweiten Teil sollen dann auf Grundlage des bekannten Romans Soll und Haben von Gustav Freytag die Differenzierungsstrategien antisemitischer Propaganda genauer untersucht werden.

\title{
Soziale Praktiken: nichtjüdische und jüdische Verbürgerlichung
}

Obwohl ihr Habitus im 19. Jahrhunderts keine Fremdheitserfahrungen oder Irritationen in der Mehrheitsgesellschaft hervorrief, blieben die akkulturierten Juden dennoch zumeist gesellschaftlich marginalisiert oder wurden sogar

9. Koschorke, „„̈̈hnlichkeit", S. 37.

10. Kimmich, „Ähnlichkeit in der Wissensgeschichte”, S. 15. 
exkludiert. Der (nicht nur) in Deutschland aufflammende Antisemitismus rekurrierte allgemein auf Alteritätserfahrungen, wenngleich in durchaus widersprüchlicher Weise: Einerseits warf man den (noch) nicht assimilierten Juden mangelnde Anpassungsbereitschaft vor, da ihr zum Teil noch stark durch religiöse Vorschriften geprägter Habitus mit den modernen, aufgeklärten Kulturpraktiken nicht kompatibel erschien; andererseits avancierte der soziale Aufstieg der bürgerlichen Schicht der Juden zum "Symbol der Schattenseiten des gesellschaftlichen und technischen Umbruchs"11, der angeblich eine zivilisatorisch-dekadente Idee von Modernität sowie Phänomene wie den Kapitalismus und die Entfremdung des Menschen vorantreibe. Hieran schloss sich nahtlos die klischeehafte und oft karikaturale Beschreibung des ökonomischen Erfolgs der Juden als Resultat von Ausbeutung und Gier an, die sich nicht nur in dezidiert antisemitischen Propagandaschriften, sondern auch in politischen, philosophischen oder wirtschaftswissenschaftlichen Schriften sowie den literarischen Werken aufgeklärter Autorinnen und Autoren finden lassen.

Ohne Zweifel kann der aggressive, direkte, ebenso wie der latente Antisemitismus im 19. Jahrhundert als eine Reaktionen auf den rasanten ökonomischen Aufstieg der jüdischen Bevölkerung zwischen 1800 und 1870 und den hohen Grad ihrer Verbürgerlichung interpretiert werden: „Man schätzt, daß gegen Ende des 18. Jahrhunderts rund 80 Prozent der in Deutschland ansässigen Juden zur untersten, ,von der Hand in den Mund' lebenden Schicht gehörten." ${ }^{12}$ Obwohl die politische Gleichstellung der Juden nach 1815 aufgrund der Restauration ins Stocken kam, waren nur siebzig Jahre später die meisten von ihnen bereits Teil des etablierten Bürgertums: „Es entstanden ein Großbürgertum und eine breite Mittelschicht, während die verarmte alte,Unterklasse' schrumpfte." ${ }^{13}$

Der „ökonomische Aufstieg [war] begleitet von einer kulturellen und sozialen Integration" ${ }^{14}$ der Juden, die vor allem auf ihrer starken Anpassungsbereitschaft beruhte. Jürgen Kocka in der Annahme folgend, dass das Bürgertum „weder als Stand [...] noch als Klasse [...], sondern als eine Kultur, als ein spezifisches Ensemble bestimmter "Normen und Werte, Lebensstile und Anschauungen"15 begriffen werden muss, bedeutet für Shulamit Volkov "Verbürgerlichung vor allem Akkulturation". ${ }^{16}$ Doch die Hürde der kulturellen Anpassung hatten alle Neuankömmlinge im Bürgertum gleichermaßen zu nehmen. Der gesellschaftliche Aufstieg verlangte von jedem eine Akkulturation, d.h. eine Aneignung der sozialen Praktiken und Gepflogenheiten des neuen Milieus, nicht allein von der

11. WernerBergmann, Geschichtedes Antisemitismus, München, C.H.Beck'scheVerlagsbuchhandlung, 42010, S. 27.

12. Shulamit Volkov, „Die Verbürgerlichung der Juden in Deutschland“, in dies., Antisemitismus als kultureller Code, München, C.H. Beck'sche Verlagsbuchhandlung, 2000, S. $111 \mathrm{f}$.

13. Bergmann, Antisemitismus, S. 36.

14. Ibid.

15. Volkov, „Verbürgerlichung der Juden“, S. 113f; vgl. hierzu auch Jürgen Kocka, Bürger und Bürgerlichkeit im 19. Jahrhundert, Göttingen, Vandenhoeck \& Ruprecht, 1987, S. 43.

16. Volkov, "Verbürgerlichung der Juden“, S. 114. Vgl. hierzu auch Daniel Azuélos, L'Entrée en bourgeoisie des Juifs allemands ou le paradigme libéral (1800-1933), Paris, PU Paris-Sorbonne, 2003. 
jüdischen Minorität. Die mitunter bittere Erfahrung, dass es leichter ist zu Geld als zu gesellschaftlichem Ansehen zu kommen, teilten die Juden insofern mit allen Aufsteigern im Bürgertum. ${ }^{17}$

AngesichtsderTatsache,dasssich dieSituationdesjüdischenundnicht-jüdischen Bürgertums in Deutschland Mitte des 19. Jahrhunderts also in vielem ähnelte, erscheint es geradezu kontrafaktisch, dass das antisemitische Bildprogramm in der Regel auf stereotype Darstellungen nichtassimilierter Juden zurückgriff. Anders als diese Diffamierungen suggerieren, glichen sich beide Bevölkerungsgruppen sowohl hinsichtlich ihrer Betätigungsfelder als auch ihres Selbstverständnisses. Ohne Hoffnung auf eine entscheidende Verbesserung der Möglichkeiten einer politischen Einflussnahme nach der gescheiterten Revolution von 1848 stand im Mittelpunkt der bürgerlichen Strategie das Bestreben, das politische Vakuum durch wirtschaftlichen Erfolg zu kompensieren. Die Kapitalisierung ging mit einer zivilisatorischer Modernisierung sowie einer weitgehenden Urbanisierung einher. „Mit zunehmender Verstädterung und der Revolutionierung von Verkehr, Transport und Kommunikation gewann der Handelskapitalismus im 19. und 20. Jahrhundert immens an Gewicht." ${ }^{18}$ Anders als die zunehmend verarmende Aristokratie, die noch lange auf vormoderne Produktionsformen wie die Landwirtschaft spezialisiert blieb, gründete sich der ökonomische Aufschwung des Bürgertums weitgehend auf der Industrialisierung und dem Warenhandel in einem städtischen Umfeld. Angesichts dieser Entwicklungen entsprach die gängige antisemitische Behauptung eines kulturellen Antagonismus zwischen geographisch verwurzelter Mehrheitsgesellschaft und mobilem Judentum bereits Mitte des 19. Jahrhunderts nicht mehr der Realität. Der wirtschaftliche Aufstieg als wesentlicher Motor der fortschreitenden Emanzipation des Bürgertums hatte zudem faktisch wenig mit Rückbesinnung auf Traditionen zu tun, im Gegenteil: Kaufleute, die Handel treiben, gleich ob es sich um Juden oder Nichtjuden handelt, können es sich nicht leisten, sich abzuschotten; sie müssen in Kontakt mit anderen Regionen und Ländern treten. Gleichwohl gab es in beiden Gruppen einen für den kapitalistischen Erfolg im 19. Jahrhundert typischen Zusammenhalt zwischen Familie, Haus und Unternehmen. ${ }^{19}$

Doch ökonomische Gewinne alleine reichen nicht aus für einen gesellschaftlichen Aufstieg. Ein ebenfalls bedeutsamer Aspekt war die akademische Bildung und Kultur, d.h. der Erwerb von symbolischem Kapital durch Anerkennung in den Bereichen Wissenschaft und Kunst, deren Bedeutung sich unter anderem im Entstehen des Bildungsbürgertums spiegelt, das in dieser Form keine Entsprechung in anderen Ländern findet. Aber auch hier gilt: Orte des wissenschaftlichen und künstlerischen Erfolgs sind die Großstädte und Metropolen, nicht die ländlichen Regionen. ${ }^{20}$ In diesem Kontext spielte auch

17. Ibid., S. 115 .

18. Jürgen Kocka, Geschichte des Kapitalismus, München, C.H. Beck'sche Verlagsbuchhandlung, 2013, S. 78.

19. Ibid., S. 86.

20. Vgl. Jürgen Kocka, „Bürger und Bürgerlichkeit im Wandel“, Aus Politik und Zeitgeschichte 9-10, 2008, S. 4. 
die deutsche Sprache für beide Gruppen gleichermaßen eine wichtige Rolle. So besaß die Hinwendung zum Hochdeutschen, das sich „erst seit der zweiten Hälfte des 18. Jahrhundert [als] eine normierte gemeinsame Sprache in Deutschland gegen eine Vielzahl von Dialekten und Mundarten durchgesetzt ${ }^{21}$ hatte, für die bürgerliche Emanzipation ein wichtige Rolle. „Die sichere Beherrschung des Hochdeutschen [...] gehörte im 18. Jahrhundert bereits fest zum kulturellen Selbstverständnis des deutschen Bürgertums." ${ }^{22}$ Gleiches gilt für die jüdische Minderheit: Shulamit Volkhov folgend, war schon zu „Beginn des 19. Jahrhunderts [...] die Ambivalenz verschwunden: Deutsch, Hochdeutsch oft in hyperkorrekter Form sollte die Sprache des jüdischen Bürgertums sein." ${ }^{\prime 23}$ Auch wenn der "Sprachwechsel vom Jiddischen zum Deutschen [...] uneinheitlich verlief" ${ }^{\prime 2}$, wurde von akkulturierten Juden das traditionelle Jiddisch und der damit verbundene Jargon vehement " ${ }_{\text {als }}$ Zeichen der Andersheit" abgelehnt. ${ }^{25}$

Angesichts der offensichtlich großen Ähnlichkeiten zwischen emanzipiertem jüdischem und nichtjüdischem Bürgertum im 19. Jahrhundert ist es im Blick auf die Entstehung einer bürgerlichen Kultur, wie Volkov bemerkt, "kein Novum", die "Geschichte der deutschen Juden als symptomatisch für den Zustand der gesamten deutschen Gesellschaft zu betrachten". ${ }^{26}$ Anders als diejenigen Juden, die weiterhin ihren orthodox-religiösen Habitus nach außen affirmierten und deutlich sichtbar anders blieben, waren die akkulturierten Juden in der nichtjüdischen Mehrheitsgesellschaft schnell fast unsichtbar geworden.

Insofern bereiteten nicht die Unterschiede, sondern die Ähnlichkeiten Probleme, zumal die anpassungswilligen Juden in relativ kurzer Zeit mehr und mehr zu erfolgreichen Konkurrenten avanciert waren. Da allenfalls noch ihre Familiennamen auf ihre Herkunft und Zugehörigkeit deuteten, war es jedoch nicht leicht, sie zu identifizieren. Die Ähnlichkeit ist daher ein wichtiger Grund, warum die Juden in antisemitischen Narrativen zu besonders gefährlichen, da unsichtbaren Feinden avancieren.

Doch es war nicht allein die Konkurrenzsituation, die zu einer Ausgrenzung, ja Stigmatisierung der Juden innerhalb der Mehrheitsgesellschaft führte, um deren Position im Wirtschaftsfeld zu schwächen. So wurde der Umbruch des ökonomischen Systems von vielen, die Schwierigkeiten hatten, sich in den neuen Verhältnissen zurechtzufinden, als Krise empfunden. Zudem brachte die wachsende ökonomische Macht des Bürgertums zahlreiche soziale Probleme und Ungerechtigkeiten für die Unterschicht mit sich, die nach einer moralischgesellschaftlichen Legitimierung der ausschließlich auf den eigenen Vorteil und Gewinn bedachten kapitalistischen Praktiken verlangte. Zur gesellschaftlichen (Neu-)Orientierung und gleichzeitiger Rechtfertigung des eigenen ökonomischen

21. Volkov, "IVerbürgerlichung der Juden", S. 118.

22. Marion Aptroot, Roland Gruschka, Jiddisch. Geschichte und Kultur einer Weltsprache, München, C.H. Beck'sche Verlagsbuchhandlung, 2010, S. $81 \mathrm{f}$.

23. Volkov, "Verbürgerlichung der Juden", S. 118.

24. Aptroot, Gruschka, Jiddisch, S. 82.

25. Volkov ,'IVerbürgerlichung der Juden", S. 118.

26. Ibid., S. 113. 
Gewinns zuungunsten vieler bot sich für das nichtjüdische Bürgertum eine „Abgrenzung bürgerlicher Wirtschaftspraktiken von einer jüdischen Praxis der Geschäfts- und Handelsformen" ${ }^{\prime 27}$ an. Dabei wurde die abstrakt und daher gefährlich erscheinende Geldwirtschaft „im Verlauf des Jahrhunderts zunehmend als eigentliche Wurzel der Übel der modernen Gesellschaft betrachtet ${ }^{\text {"28 }}$ und den Juden zugeschrieben. So gelten die spekulativen Operationen an der Börse als "fragwürdige jüdische ,Luftgeschäfte ${ }^{\prime \prime 2}{ }^{29}$, wohingegen sich die nichtjüdischen Tätigkeiten angeblich allein auf den Handel mit konkreten Waren konzentrierte. Die bekannte „Interferenzzone zwischen antikapitalistischer und antisemitischer Semantik" 30 mündet auf diese Weise in eine deutliche Gegenüberstellung von jüdischer und "deutscher Arbeit" ${ }^{\prime 31}$ :

\begin{abstract}
,Deutsche Arbeit' erscheint dabei nicht als eine beliebige nationale Tugend neben anderen, die den äußeren und inneren Feinden der Nation abgesprochen wird, dient also nicht allein der Definition und Demarkation nationaler Identität. [Sie] erscheint [...] darüber hinaus als Form konkreter Vermittlung und somit als konstitutiv für die Herstellung von Gemeinschaft. Sie fungiert [...] als wichtigstes Mittel gegen die gemeinschaftszersetzenden Tendenzen der kapitalistischen Gesellschaft, gegen die Bedrohung, die der Form der Nation durch eben jene Moderne erwächst, die sie doch erst hervorbringt. ${ }^{32}$
\end{abstract}

In diesem Sinne muss der Diskurs von der "deutschen Arbeit", nicht nur als gesellschaftliche Legitimierungsstrategie, sondern politisches Programm verstanden werden, das auf die Konstruktion einer Opposition zwischen jüdischer und nichtjüdischer Arbeit setzt, um die evidenten Ähnlichkeiten bewusst zu unterminieren.

\title{
Sein und Schein - Soll und Haben: Der Roman als politisches Programm
}

Ein frühes und besonders anschauliches Beispiel für die beschriebene politische Strategie liefert der 1855 erschienene Roman Soll und Haben von Gustav Freytag, der zu den erfolgreichsten deutschsprachigen Romanen aller Zeiten zählt und

27. Sabina Becker, „Erziehung zur Bürgerlichkeit: Eine kulturgeschichtliche Lektüre von Gustav Freytags Soll und Haben", in Florian Krobb (Hrsg.), 150 Jahre ,Soll und Haben'. Studien zu Gustav Freytags kontroversem Roman, Würzburg, Königshausen \& Neumann, 2005, S. 38.

28. Christine Achinger, „,Selbstzerstörung des Liberalismus bei Gustav Freytag“, in Nicolas Berg (Hrsg.), Kapitalismusdebatten um 1900 - über antisemitisierende Semantiken des Jüdischen, Leipzig, Leipziger Universitätsverlag, 2011, S. 376.

29. Franziska Schößler, „Blutzauber, Magie und Spekulation. Die unproduktiven Wirtschaftspraktiken im ,Jüdischen Kaufhaus'", in Nicole Colin, Franziska Schößler (Hrsg.), Das nennen Sie Arbeit! Der Produktivitätsdiskurs und seine Ausschlüsse, Heidelberg, Synchron, 2012, S. 68.

30. Benno Wagner, „Verklärte Normalität. Gustav Freytags Soll und Haben und der Ursprung des ,Deutschen Sonderwegs' ${ }^{\prime \prime}$, Internationales Archiv für Sozialgeschichte der deutschen Literatur 30 (2), S. 15.

31. Vgl.Wilhelm Heinrich Riehl, Die deutsche Arbeit, Stuttgart/Berlin, Cotta'scheVerlagsbuchhandlung, 1861.

32. Christine Achinger, "Selbstzerstörung des Liberalismus", S. 368. 
bis weit in die 1960er Jahre hinein ungeheure Auflage- und Verkaufszahlen erreichte. ${ }^{33}$ Bereits direkt nach seiner Veröffentlichung wurde das in Breslau zwischen dem Vormärz und der Revolution von 1848 spielende Buch aufgrund seiner exzessiven Verwendung antisemitischer Stereotype heftig kritisiert. ${ }^{34}$ Heute herrscht allgemeiner Konsens darüber, dass es sich um einen Fall von literarischem Antisemitismus handelt. ${ }^{35}$

Soll und Haben gilt als Musterroman des programmatischen Realismus und erscheint hier vor allem hinsichtlich seines dezidiert didaktischen Charakters interessant, der ihn zu einer Art bürgerlicher Lehrfibel macht. Der Roman liefert keineswegs eine realistisch überzeugende Darstellung oder gar Analyse der herrschenden Verhältnisse, ${ }^{36}$ sondern bietet vielmehr, ganz im Sinne des programmatischen Realismus, eine klar ausformulierte politische Handlungsanleitung, eine Gebrauchsanweisung, die dem Leser darüber Auskunft erteilen will, wie er sich zu verhalten und vor allem auf welche Weise er seine Geschäfte zu führen hat, um die Verwirklichung der bürgerlichen Gesellschaft nach der gescheiterten Revolution dennoch voranzutreiben. Das Soll steht also nicht nur im Titel an erster Stelle.

Aus diesem Grund wäre es auch verfehlt, den Roman als historische Quelle zu nutzen, um Informationen über die gesellschaftliche Situation der Juden in Breslau Mitte des 19. Jahrhunderts zu erhalten. Verstanden als Entwurf eines politisch anzustrebenden Ideals, in dessen Mittelpunkt das Konstrukt eines deutschen nationalen bürgerlichen Kapitalismus steht, der sich zwar nicht sichtbar, angeblich aber doch entscheidend von seiner jüdischer Variante unterscheidet, muss Soll und Haben vielmehr als politisches, in die Zukunft weisendes Programm gelesen werden, das in einigen Punkten die realen Verhältnisse sogar bewusst konterkariert. Ausgehend von dem eingangs beschriebenen Konzept der Ähnlichkeit, wird daher im Folgenden gezeigt, auf welche Weise Freytag die Andersartigkeit sozialer Praktiken des jüdischen akkulturierten Bürgertums konstruiert und Differenzen zu der sich faktisch kaum unterscheidenden nichtjüdischen Mehrheitsgesellschaft inszeniert. ${ }^{37}$

33. „[N]ach fünf Jahren waren bereits 22000 Bücher verkauft; T.E. Carters Hochrechnungen gehen von insgesamt ca. 1222000 verkauften Exemplaren zwischen 1855 und 1965" aus. Vgl. Florian Krobb, „Einleitung: Soll und Haben nach 150 Jahren“, in ders. (Hrsg.), 150 Jahre ,Soll und Haben', S. 9. Selbst nach dem Zweiten Weltkrieg wurde der Roman noch intensiv rezipiert - zumindest in Westdeutschland. So übertrafen die Absatzzahlen hier zwischen 1950 und 1965 mit 420000 Exemplaren sogar noch die zwischen 1915 und 1930. Vgl. Dirk Oschmann, "Einleitung“, in HansWerner Hahn, Dirk Oschmann (Hrsg.), Gustav Freytag (1816-1895). Literat - Publizist - Historiker, Köln/ Weimar/ Wien, Böhlau, 2016, S. 8.

34. Vgl. Till van Rahden, Juden und andere Breslauer: die Beziehungen zwischen Juden, Protestanten und Katholiken in einer deutschen Großstadt von 1860 bis 1925, Göttingen, Vandenhoeck \& Ruprecht, 2000, S. 275.

35. Dies wurde 2005 noch einmal zusammenfassend in dem bereits zitierten Sammelband bestätigt: Florian Krobb (Hrsg.), 150 Jahre,Soll und Haben'.

36. Vgl. Becker, "Erziehung zur Bürgerlichkeit", S. 39.

37. Anzumerken ist, dass, wenngleich die Juden im Zentrum dieser Betrachtung stehen, diese nicht die einzigen Opfer stereotyper Überspitzung sind. Auch das Verhalten von Polen, Amerikanern 
Im Mittelpunkt der Geschichte steht der aus der Kleinstadt Ostrau stammende Anton Wohlfart, der im Kontor von Traugott Schröter in der nahe gelegenen Hauptstadt eine Ausbildung beginnt. Gegenfigur zu Anton ist Veitel Itzig, ein ehemaliger jüdischer Schulkamerad, der zur gleichen Zeit von dem assimilierten jüdischen Kaufmann Hirsch Ehrenthal eingestellt wird. Ausgehend von dieser ähnlichen Ausgangsposition schlagen die beiden jungen Männer jeweils grundlegend andere Wege ein. Am Schluss ist Veitel Itzig durch wenig ehrenhafte Geschäfte zwar zu Geld gekommen, begeht aber schließlich einen Mord im Affekt und ertrinkt bei einem Fluchtversuch. Anton findet hingegen sein Glück in der Arbeit: Er wird von Herrn Schröter in die Geschäftsführung aufgenommen und verlobt sich mit dessen Schwester.

Die Struktur dieser Versuchsanordnung, d.h. die zeitliche und räumliche Parallelität der Lebenswege der beiden Schulkameraden, die zur gleichen Zeit in der gleichen Stadt landen, erlaubt es, diese bis ins Detail hinein zu vergleichen. Viele Analysen von Soll und Haben stellen dabei das Judentum als Gegenwelt zum deutschen Bürgertum in den Mittelpunkt. ${ }^{38}$ Dies erscheint angesichts der deutlichen Ähnlichkeiten der jüdischen und nichtjüdischen Lebenswelt jedoch zu kurz gegriffen. Wie Christine Achinger bemerkt, ist für

\begin{abstract}
die Konstruktion des Gegensatzes zwischen Deutschen und Juden in Soll und Haben [...] entscheidend, dass dieser eben nicht in jeder Hinsicht ein polarer ist. Deutsche Bürger wie Juden sind Kaufleute [...] bzw. Händler [...], beide ernähren sich durch disziplinierte Arbeit, und beide Gruppen gehören der modernen Gesellschaft an. Hervorgehoben wird durch diese Figurenkonstellation gerade die Differenz in der Gleichheit; erst der parallele Verlauf ihrer Karriere macht Veitel und Anton zu Gegenspielern. Und erst durch diese Konstruktion werden jüdische und deutsche Daseinsweise zu alternativen Varianten derselben modernen Existenz. ${ }^{39}$
\end{abstract}

Die Konkurrenzsituation zwischen Hirsch Ehrenthal und dem nichtjüdischen Kaufmannshaus Schröter wird auf allen Ebenen durchdekliniert. Nicht nur in ökonomischer Hinsicht führt Freytag die (angeblich) unterschiedlichen Handelsformen und ihren Erfolg an Fallbeispielen vor, auch die Lebensstile und die äußere Erscheinung der Frauen konkurrieren in dem vorgestellten Programmentwurf bürgerlicher Kultur. Die im Vergleich aufscheinenden ,feinen Unterschiede' der sozialen Praktiken der beiden Handelsfamilien erscheinen bei genauer Betrachtung allerdings als ein in vielen Punkten von der Realität abweichendes Konstrukt, das offensichtlich dazu dienen soll, den Leser trotz der Ähnlichkeiten für die (angeblich) profunden Unterschiede zu sensibilisieren. So fällt beim Vergleich der Beschreibungen der Frauen der jüdischen und nichtjüdischen Kaufmannsfamilie auf, dass hier zahlreiche

sowie des Adels dient als Negativfolie, um in Abgrenzung hierzu das Ideal des bürgerlichen Arbeitsethos zu veranschaulichen.

38. Vgl. hierzu Dagmar C.G. Lorenz, „Ein Roman für zwei Jahrhunderte: Freytags Stereotypen bis zur Jahrtausendwende“, in Florian Krobb (Hrsg.), 150 Jahre Soll und Haben, S. 302: „Jüdische Gestalten sind dagegen ausschließlich Träger negativer Eigenschaften - Opportunismus, parasitärer Materialismus, unschöpferisch-dilettantische Gelehrsamkeit und Körperschwäche. Sie stellen eine Bedrohung für die sich anbahnende deutsche Ordnung dar."

39. Achinger, ,"Selbstzerstörung des Liberalismus", S. 369. 
Parallelen hervorstechen: So entsprechen die Frau und Tochter Ehrenthals zwar auf den ersten Blick beide dem Klischee der ,schönen Jüdin', gleichwohl gibt es aber viele Ähnlichkeiten: Sowohl Frau Ehrenthal als auch Sabine Schröter ${ }^{40}$ haben beide „rabenschwarzes" Haar und der Assimilationsprozess der jüdischen Familie scheint so weit fortgeschritten, dass die attraktive Tochter Ehrenthals auch nur noch eine "sehr wenig gebogene" Nase hat. ${ }^{41}$

Da die äußeren Kennzeichen der Herkunft, wie hier deutlich wird, kaum sichtbar sind, konzentriert sich Freytag auf die Konstruktion einer Differenz durch eine sprachliche Markierung: Alle jüdischen Figuren, außer der Ausnahmeexistenz Bernhard Ehrenthal, sprechen ein falsches Deutsch, wenngleich dies, wie oben beschrieben, keinesfalls dem Habitus des jüdischen akkulturierten Bürgertum entspricht. Selbst der Sprachduktus des jungen Veitel Itzig, der noch ganz als polnischer "Jude von altem Styl" ${ }^{42}$ beschrieben wird, ist unrealistisch, insofern dieser ja gemeinsam mit Anton dieVolkschule besucht hat. Gänzlich kontrafaktisch erscheint dann aber die Tatsache, dass der akkulturierte und erfolgreiche Hirsch Ehrenthal nicht von seiner falschen Wortstellung ablassen will. Im Roman dient die sprachliche Kennzeichnung der jüdischen Figuren vor allem ihrer besseren Wahrnehmbarkeit. Gleichzeitig werden sie hierdurch aber auch grundsätzlich aus Freytags nationalistischem Programm des deutschen Bürgertums ausgegrenzt.

Doch kommen wir zum eigentlichen Kernthema: Ziel des Romans ist es, über die sichtbare äußere Erscheinung und Sprache hinaus eine Abgrenzung der deutschen zur jüdischen Arbeit zu konstruieren, d.h. zu zeigen, "dass das ökonomische und wirtschaftliche Agieren des Bürgertums sich strikt von einem jüdischen Materialismus unterscheidet" ${ }^{\prime 3}$. Auf der Negativfolie der durchaus nicht immer unredlichen Geschäfte des bürgerlichen, emanzipierten und akkulturierten Juden Hirsch Ehrenthal und seiner Familie wird gezeigt, in welcher Weise man sich zu verhalten bzw. nicht zu verhalten hat, um die politische Zukunft des deutschen Bürgertums auf der Basis einer moralischen Veredelung der kapitalistischen Kulturtechniken voranzutreiben.

Die Besonderheiten der nichtjüdischen Arbeit zeigen sich anschaulich in der Gegenüberstellung der nachhaltigen, konkreten Ansammlung von Waren, die im Hause Schröter mit eigenem Gelde „gekauft [...] und auf eigene Gefahr" ${ }^{\prime 4}$ vorrätig gehalten werden und der flüchtigen Spekulation bzw. einer Konzentration auf abstrakte Geldwerte auf jüdischer Seite. Ehrenthal geht es immer nur um das Geld an sich und er hat keinerlei Probleme damit, "Schulden [zu] machen, um reich

40. „Fräulein Sabine zeigte über dem eleganten Sommerkleide ein feines bleiches Gesicht, von rabenschwarzem Haar eingefaßt." (Freytag, Soll und Haben, S. 61).

41. "Madame Ehrenthal war eine volle Frau in schwarzer Seide, mit starken Augenbrauen und rabenschwarzen Hängelocken [...]. Ihre Tochter aber war in der Tat eine Schönheit, eine große edle Gestalt mit glänzenden Augen, dem reinsten Teint und einer nur sehr wenig gebogenen Nase." (Freytag, Soll und Haben, S. 50).

42. [Gustav Freytag], „Die Juden in Breslau“, Die Grenzboten. Zeitschrift für Politik, Literatur und Kunst 8 (2), Bd. 2, 1849, S. 145.

43. Becker, "Erziehung zur Bürgerlichkeit”, S. 36.

44. Freytag, Soll und Haben, S. 56. 
zu werden"45. Die Waren dienen lediglich als Mittel zum Zweck. Im Unterschied dazu stehen in der Beschreibung des Handelshauses Schröter immer die Dinge, die Objekte sichtbar im Vordergrund. Das Geschäft der Familie Schröter wird als „altertümlich, solid und großartig“ beschrieben, es ist „ein Warengeschäft, wie sie jetzt immer seltener werden ${ }^{\prime 46}$ angesichts der Entfremdung der Arbeit und der Auflösung des konkreten Bezugs zu den Gegenständen. Die Gefahren der neuen Zeit können prinzipiell zwar nicht aufgehalten werden - „jetzt, wo Eisenbahnen und Telegraphen See und Inland verbinden, wo jeder Kaufmann aus den Seestädten durch seine Agenten die Waren tief im Lande verkaufen läßt, fast bevor sie im Hafen angelangt sind" ${ }^{47}$. Schröter gibt jedoch ein Beispiel dafür, wie man als Bürger mithilfe einer inneren Abwehrhaltung diesen zivilisatorischen Prozess unterlaufen kann, um Sicherheit und Gemeinschaft zu schaffen. So hat sein „alte[s] weltbekannte[s] Binnengeschäft ein stolzes, ja fürstliches Ansehen, und was mehr wert ist, es war ganz gemacht, bei seinen Teilhabern feste Gesinnung und ein sicheres Selbstgefühl zu schaffen".48

Die angeblich profunden Unterschiede lassen sich jedoch von außen nicht erkennen und müssen folglich auf andere Weise veranschaulicht werden. Im Zentrum der Beschreibung steht daher die „intrinsische Motivation“ der Figuren, d.h. die Frage „ob man Reichtum erwirtschaften oder ein erfülltes Leben führen will", insofern "gute Arbeit" nicht nur "ökonomisch sinnvoll", sondern auch „moralisch korrekt" zu sein hat. ${ }^{49}$ Bereits sechs Jahre vor dem Erscheinen des Romans hatte Freytag in dem anonym erschienenen Artikel "Die Juden in Breslau“ in der Zeitschrift Die Grenzboten explizit auf den für ihn essentiellen Unterschied zwischen deutscher und jüdischer Arbeit hingewiesen:

Jene Tugend, durch welche der solide Kaufmann seinen Egoismus adelt, die kaufmännische Ehre, er [der Jude] kennt sie nicht, aber er nimmt den Schein der Sicherheit und Zuverlässigkeit mit Ausdauer und Erfolg so lange an, bis es ihm lohnt, irgendetwas bedenkliches zu thun, was nach unsern Begriffen durchaus unehrlich ist ihm nur einen gewagten Streich gilt. In seinen Geschäften mit soliden Handlungen spekuliert er mit seiner Ehrlichkeit ebenso fein als naiv. ${ }^{50}$

Die kapitalistische Rechtfertigungsstrategie ist hier klar zu erkennen: Der solide nichtjüdische bzw. christliche Kaufmann legitimiert seinen Egoismus durch seine "kaufmännische Ehre", die der Jude nicht kennt und offenbar auch, aller Akkulturation zum Trotz, nicht lernen kann. Laut Gustav Freytag weiß der (nichtjüdische) Kaufmann dieses „Ehrgefühl“ von "gesetzlicher Ehrenhaftigkeit sehr genau zu unterscheiden" ${ }^{\prime 1}$, wobei das fehlende Ehrgefühl die jüdischen Kaufleute nicht daran hindert, unter Umständen ehrbar zu arbeiten. Allerdings ist das ehrenhafte Verhalten bei jüdischen Kaufleuten nichts als schöner Schein, der

45. Ibid., S. 35.

46. Ibid., S. 56 .

47. Ibid.

48. Ibid.

49. Dirk Oschmann, „Der Streit um die Arbeit. Gustav Freytag Soll und Haben“, in Hans-Werner Hahn, Dirk Oschmann (Hrsg.), Gustav Freytag, S. 131f.

50. Gustav Freytag, „Die Juden in Breslau“, S. 146.

51. Ibid. 
sogar seinerseits zum Spekulationsobjekt werden kann, wenn jüdische Kaufleute mittels Imitation ihre Ehrlichkeit inszenieren, um daraus Vorteile zu schlagen. Die Trennungslinie zwischen deutscher und jüdischer Arbeit befindet sich also im Spannungsfeld von Sein und Schein, ein Gegensatz, der in Soll und Haben als unaufhebbar gezeichnet wird. Dabei werden auch hier die Unterschiede kunstvoll gegen die naheliegende Feststellung einer großen Ähnlichkeit konstruiert: Der Name Hirsch Ehrenthal konterkariert insofern nicht Freytags Behauptung, die Juden hätten kein Ehrgefühl, vielmehr ist die Ehre, die der jüdische Kaufmann im Namen trägt nichts als schöner Schein. Der akkulturierte Jude ist dem nichtjüdischen Kaufmann zwar äußerlich ebenbürtig, moralisch jedoch keinesfalls auf gleicher Höhe wie Traugott Schröder.

Narrationstechnisch verdichtet Freytag die unaufhebbare Differenz von Sein und Schein mithilfe der Präsenz eines allwissenden Erzählers. Dieser räumt in seinen Beschreibungen und Kommentaren der Gedankenwelt der Figuren einen zentralen Platz ein, um so ihre (unsichtbare) Motivation und geheimsten Wünsche ans Licht zu bringen, welche in den Handlungen selbst nicht unbedingt sichtbar werden. ${ }^{52}$ Bereits in der ersten Nacht in der fremden Stadt wird der Unterschied zwischen nichtjüdischem und jüdischem Kapitalismus, d.h. konkretem Warenhandel und windigem Geldgeschäft beispielhaft anhand der Träume von Anton und Veitel vorgeführt: Während Anton "sich selbst auf einem großen Warenballen sitzen und durch die Luft fliegen" sieht, entdeckt Veitel Itzig „mit Behagen, daß er ein Baron geworden war, welcher von Hirsch Ehrenthal um ein Almosen angeredet wurde"..$^{53}$ Anton geht es um die Dinge, Veitels Gedanken kreisen ausschließlich um Geld und Status und dies zudem in Verbindung mit einem geradezu abgründig unmoralischen Charakter, wie Freytag in der sich anschließenden Reaktion Veitels demonstriert, der träumt, Ehrenthal sechs Dukaten als Geschenk zu geben: „Über diese Großmut erschrak er im Traume so, daß er mit Beinen und Händen um sich schlug. ${ }^{\prime 4}$

Die Traum-Gegenüberstellung ist nur ein Beispiel dafür, wie Freytag die Fokussierung auf die Motivation und die moralischen Überzeugungen der Protagonisten nutzt, um den Leser zu sensibilisieren, die scheinbar nur feinen Unterschiede als essentielle Gegensätze zu begreifen. Das so entstehende Alteritätskonstrukt bietet die ideale Grundlage einer politisch-moralischen Rechtfertigungsstrategie, welche die Beteiligung des Bürgertums an der als jüdisch verschrienen kapitalistischen Ausbeutung legitimiert.

52. Insofern dominiert hier mehr der Wunsch nach Innerlichkeit als Realismus oder, wie es Hans Mayer formulierte: „Gustav Freytag half mit, eine Welt der ,machtgestützten Innerlichkeit' (um den späteren Ausdruck Thomas Manns zu gebrauchen) zu etablieren und zu verklären." (Hans Mayer, "Gustav Freytags bürgerliches Heldenleben", in Freytag, Soll und Haben, S. 839).

53. Freytag, Soll und Haben, S. 55.

54. Ibid. 


\section{Fazit}

Anhand einiger Beispiele aus dem Roman Soll und Haben, der hier als bürgerliche Lehrfibel gelesen wurde, konnte gezeigt werden, dass eine Konzentration auf die Kategorien Identität und Alterität zu kurz greift, um das Verhältnis zwischen nichtjüdischem und jüdischem Bürgertum in Deutschland im 19. Jahrhundert zu verstehen. Die methodische Hinwendung zum,vagen' Begriff der Ähnlichkeit ermöglicht hingegen über die konstruierten Grenzen hinweg, die emanzipierten Juden und nichtjüdischen bürgerlichen Aufsteiger zunächst einmal als eine gesellschaftliche Gruppe zu begreifen, die sich gleichermaßen den Anforderungen der Akkulturation zu stellen hatte. Aufgrund des bedeutsamen ökonomischen Strukturwandels und des damit verbundene Rechtfertigungsdrucks, sich als Gewinner in einem Ausbeutungssystem zu legitimieren, entwickelte sich Mitte des 19. Jahrhunderts ein symbolischer Abwehrdiskurs gegenüber der gut integrierten, aber minoritären Gruppe der emanzipierten Juden. Angesichts der zu konstatierenden Ähnlichkeiten und der Tatsache, dass das jüdische Bürgertum „seit Beginn des 19. Jahrhunderts mit seinen soziokulturellen Bestrebungen doch eigentlich zur bürgerlichen Kerngruppe gehörte, mithin sehr wohl bürgerliche Prinzipien verinnerlicht, ja mit formuliert hatte ${ }^{\prime 55}$, kann die Gegenüberstellung deutscher und jüdischer Arbeit als ein kontrafaktisches Konstrukt bezeichnet werden.

55. Andrea Hopp, "Gustav Freytag und die Juden“, in Hans-Werner Hahn, Dirk Oschmann (Hrsg.), Gustav Freytag, S. 237. 
